May, 1995

BGU-95 / 10 / May - PH

hep-th/9505128

\title{
Volume-Preserving Diffeomorphisms' versus Local Gauge Symmetry
}

\author{
E.I. Guendelman, E. Nissimov ${ }^{1,2}$ and S. Pacheva円巴 \\ Department of Physics, Ben-Gurion University of the Negev \\ Box 653, IL-84105 Beer Sheva, Israel \\ e-mail: guendel@bguvm.bgu.ac.il, emil@bgumail.bgu.ac.il, svetlana@bgumail.bgu.ac.il
}

\begin{abstract}
We present a new form of Quantum Electrodynamics where the photons are composites made out of zero-dimensional scalar "primitives". The rôle of the local gauge symmetry is taken over by an infinite-dimensional global Noether symmetry - the group of volume-preserving (symplectic) diffeomorphisms of the target space of the scalar primitives. Similar construction is carried out for higher antisymmetric tensor gauge theories. Solutions of Maxwell's equations are automatically solutions of the new system. However, the latter possesses additional non-Maxwell solutions which display some interesting new effects: (a) a magneto-hydrodynamical analogy, (b) absence of electromagnetic self-energy for electron plane wave solutions, and (c) gauge invariant photon mass generation, where the generated mass is arbitrary.
\end{abstract}

\footnotetext{
${ }^{1}$ On leave from the Institute of Nuclear Research and Nuclear Energy, Boul. Tsarigradsko Chausee 72, BG-1784 Sofia, Bulgaria; e-mail: emil@bgearn.bitnet, svetlana@bgearn.bitnet

${ }^{2}$ Supported in part by Bulgarian NSF grant $P h-401$.
} 


\section{Introduction}

Systems with infinite number of conservation laws have been extensively studied, because their high symmetry allows to extract non-perturbative information and in some instances, e.g. for the completely integrable two-dimensional field-theoretic models, even to solve them exactly [1]. It is however hard to find a realistic field theory in $D=4$ space-time dimensions, that allows infinite number of nontrivial conserved charges.

In this work, we will study a theory which allows an infinite number of conservation laws and which is at the same time a theory resembling very much the most respectable and most tested field theory that we know up to date - Quantum Electrodynamics (QED). This is achieved through the substitution of the ordinary local gauge symmetry of QED (which does not lead to conservation laws, but rather to constraints on the physical degrees of freedom) by a global infinite-dimensional Noether symmetry group - the group of volume-preserving (symplectic) diffeomorphisms (see, e.g. [2]). An infinite-dimensional abelian group, corresponding to the Cartan subalgebra of the group of volume-preserving diffeomorphisms, has already been identified as a symmetry of a variant of the model studied here by one of us [3].

The above mentioned Noether symmetry group acts as a group of transformations on a set of primitive dimensionless scalar fields taking values in some smooth manifold ("target" space) and preserves the volume form there. The gauge potential and the gauge field strength (the photons) in such a theory are not fundamental fields, but rather they are composites of these primitive scalar constituents. Under a volume-preserving diffeomorphism transformation, the composite gauge field is transformed by the addition of a total gradient while the composite field strength is, of course, a volume-preserving-diffeomorphism-invariant quantity. It is possible to couple the composite gauge field to matter fields in the well known minimal way so that the gradient, that is added to the gauge field under a volume-preserving transformation on the scalar constituents, is canceled by an appropriate phase transformation of the matter fields. If these matter fields are taken to be Dirac fermion fields, we obtain a "mini-QED" model that resembles very much the usual QED describing the interaction of electromagnetic field with Dirac particles. Furthermore, if the number of the primitive scalars is greater or equal to $D$ (dimension of space-time), any solution of the usual Maxwell's equations can be expressed (in a non-unique way, in general) through the primitive scalars and it is automatically a solution of mini-QED equations of motion.

However the mini-QED model does not coincide completely with QED, since it possesses solutions that do not respect Maxwell's equations. These additional solutions describe new effects that do not appear in the usual formulation of electrodynamics. Among these new features we can notice at least the following: (a) a magneto-hydrodynamical analogy, (b) absence of electromagnetic self-energy for electron plane wave solutions, and (c) generation of gauge invariant photon mass. In addition, as it was already observed in a particular variant of the mini-QED model [3], the idea of substituting local gauge invariance with a global infinite-dimensional symmetry group can be applied to the construction of models with chiral invariance which are totally anomaly free (provided the number primitive scalars is smaller than the dimension of space-time).

The above construction is carried out here also for higher antisymmetric tensor ("p- 
form") gauge theories (see, e.g. [4]), for instance - the Kalb-Ramond "2-form" gauge model [5], where the ordinary local "p-form" tensor gauge invariance is substituted with the global infinite-dimensional Noether symmetry of volume-preserving diffeomorphisms on the $p+1$ dimensional target space of primitive scalar constituents. Here again, in general, all solutions of the ordinary tensor gauge theories are automatically solutions (as composites made out of the primitive scalar constituents) to the "mini-tensor-gauge" theories, however, the latter possess additional solutions absent from the usual $p$-form gauge models.

\section{Groups of Volume-Preserving Diffeomorphisms}

First, let us recall the basic notions connected with groups of symplectic diffeomorphisms and more generally - the groups of volume-preserving diffeomorphisms on smooth manifolds (see, e.g. [2]). Let $\mathcal{T}^{2 n}$ be a (2n-dimensional) symplectic manifold with a symplectic structure which can always (at least locally) be represented in terms of a canonical constant antisymmetric $2 n \times 2 n$ matrix:

$$
\left\|\omega_{a b}\right\|=\left(\begin{array}{ccccc}
0 & 1 & 0 & 0 & \ldots \\
-1 & 0 & 0 & 0 & \ldots \\
0 & 0 & 0 & 1 & \ldots \\
0 & 0 & -1 & 0 & \ldots \\
\vdots & \vdots & \vdots & \vdots & \ddots
\end{array}\right)
$$

and let $\left\{\Phi^{a}\right\}_{a=1}^{2 n}$ denote the corresponding (local) coordinates on $\mathcal{T}^{2 n}$. Then the infinitedimensional group SDiff $\left(\mathcal{T}^{2 n}\right)$ of symplectic diffeomorphisms $\Phi^{a} \rightarrow G^{a}(\Phi)$ on the manifold $\mathcal{T}^{2 n}$ and the associated infinite-dimensional Lie algebra $\mathcal{S D}$ iff $\left(\mathcal{T}^{2 n}\right)$ of infinitesimal symplectic diffeomorphisms $G^{a}(\Phi) \approx \Phi^{a}+\omega^{a b} \frac{\partial \Gamma}{\partial \Phi^{b}}$ are defined as follows:

$$
\begin{array}{r}
\operatorname{SDiff}\left(\mathcal{T}^{2 n}\right) \equiv\left\{\Phi^{a} \rightarrow G^{a}(\Phi) ; \omega_{c d} \frac{\partial G^{c}}{\partial \Phi^{a}} \frac{\partial G^{d}}{\partial \Phi^{b}}=\omega_{a b}\right\} \\
\mathcal{S D} i f f\left(\mathcal{T}^{2 n}\right) \equiv\left\{\Gamma(\Phi) ;\left[\Gamma_{1}, \Gamma_{2}\right] \equiv\left\{\Gamma_{1}, \Gamma_{2}\right\}=\omega^{a b} \frac{\partial \Gamma_{1}}{\partial \Phi^{a}} \frac{\partial \Gamma_{2}}{\partial \Phi^{b}}\right\}
\end{array}
$$

As seen from eq.(3), the Lie-commutator is nothing but the canonical Poisson bracket on $\mathcal{T}^{2 n}$ with $\omega^{a b}$ indicating the inverse matrix w.r.t. $\omega_{a b}$ (11).

In the simplest case $n=1, \omega_{a b}=\varepsilon_{a b}, \varepsilon_{12}=1$, and $\mathcal{S D}$ iff $\left(\mathcal{T}^{2}\right)$ is precisely the algebra of area-preserving diffeomorphisms on a two-dimensional manifold $\mathcal{T}^{2}$, also known as $w_{\infty^{-}}$ algebra when $\mathcal{T}^{2}$ is a cylinder (for a review, see [6] and references therein), which contains as a subalgebra the centerless conformal Virasoro algebra. For example, in the case of torus $\mathcal{T}^{2}=S^{1} \times S^{1}$ the Lie-algebra elements of $\mathcal{S D}$ iff $\left(S^{1} \times S^{1}\right)$ are given by:

$$
\Gamma(\Phi)=\sum_{\vec{n}} \gamma_{\vec{n}} \Gamma_{\vec{n}}(\Phi) \quad, \quad \Gamma_{\vec{n}}(\Phi) \equiv \exp \{\vec{n} . \vec{\Phi}\} \quad, \vec{n}=\left(n_{1}, n_{2}\right)
$$

where $\left(n_{1}, n_{2}\right)$ are arbitrary integers. Furthermore, $\mathcal{S} \mathcal{D}$ iff $\left(S^{1} \times S^{1}\right)$ allows for a non-trivial central extension [7] which in the basis $\left\{\Gamma_{\vec{n}}\right\}$ reads:

$$
\left[\Gamma_{\vec{n}}, \Gamma_{\vec{m}}\right]=-\left(n_{1} m_{2}-n_{2} m_{1}\right) \Gamma_{\vec{n}+\vec{m}}-\vec{c} \cdot \vec{n} \delta_{\vec{n}+\vec{m}, 0}
$$


where $\vec{c}=\left(c_{1}, c_{2}\right)$ denotes the pair of "central charges".

It is well known that the group $\operatorname{SDiff}\left(\mathcal{T}^{2 n}\right)$ (2) is a subgroup of the group $\operatorname{Diff}_{0}\left(\mathcal{T}^{2 n}\right)$ of volume-preserving diffeomorphisms on the manifold $\mathcal{T}^{2 n}$. Moreover, the group $\operatorname{Diff}\left(\mathcal{T}^{s}\right)$ exists for manifolds of arbitrary (not necessarily even) dimension $s$ :

$$
\operatorname{Diff}_{0}\left(\mathcal{T}^{s}\right) \equiv\left\{\Phi^{a} \rightarrow G^{a}(\Phi) ; \varepsilon_{b_{1}, \ldots, b_{s}} \frac{\partial G^{b_{1}}}{\partial \Phi^{a_{1}}} \cdots \frac{\partial G^{b_{s}}}{\partial \Phi^{a_{s}}}=\varepsilon_{a_{1}, \ldots, a_{s}}\right\}
$$

Indeed, it is straightforward to verify that $G^{a}(\Phi)$ defined in (2) preserves the volume-form $\frac{1}{s !} \varepsilon_{a_{1} \ldots a_{s}} d \Phi^{a_{1}} \wedge \ldots \wedge d \Phi^{a_{s}}$ on $\mathcal{T}^{s}$ where the simple relation was used (for $s=2 n$ ) :

$$
\omega_{\left[a_{1} a_{2}\right.} \cdots \omega_{\left.a_{s-1} a_{s}\right]}=\varepsilon_{a_{1} \ldots a_{s}}
$$

and the square brackets indicate total antisymmetrization of indices. Accordingly, the Lie algebra $\mathcal{D} i f f_{0}\left(\mathcal{T}^{s}\right)$ of infinitesimal volume-preserving diffeomorphisms is given by:

$$
\begin{array}{r}
\mathcal{D i f f}_{0}\left(\mathcal{T}^{s}\right) \equiv\left\{\Gamma^{a}(\Phi) \quad ; \quad G^{a}(\Phi) \approx \Phi^{a}+\Gamma^{a}(\Phi), \frac{\partial \Gamma^{b}}{\partial \Phi^{b}}=0\right\} \\
\text { i.e. } \quad \Gamma^{a}(\Phi)=\frac{1}{(s-2) !} \varepsilon^{a b c_{1} \ldots c_{s-2}} \frac{\partial}{\partial \Phi^{b}} \Gamma_{c_{1} \ldots c_{s-2}}
\end{array}
$$

\section{Field-Theory Models}

Now, let us consider a set of $2 n$ (zero-dimensional) scalar fields $\left\{\Phi^{a}(x)\right\}_{a=1}^{2 n}$ on ordinary Minkowski space-time taking values in the symplectic manifold $\mathcal{T}^{2 n}$. Then, the canonical symplectic closed two-form $\Omega$ on $\mathcal{T}^{2 n}$ naturally leads to the construction of an antisymmetric tensor field $F_{\mu \nu}(\Phi(x))$ on space-time satisfying the Bianchi identity and, therefore, allowing for a potential $A_{\mu}(\Phi(x))$ :

$$
\begin{array}{r}
\Omega=\frac{1}{2} \omega_{a b} d \Phi^{a} \wedge d \Phi^{b}=\frac{1}{2} F_{\mu \nu}(\Phi) d x^{\mu} \wedge d x^{\nu} \\
F_{\mu \nu}(\Phi)=\omega_{a b} \partial_{\mu} \Phi^{a} \partial_{\nu} \Phi^{b} \quad, \quad \partial_{\kappa} F_{\lambda \mu}(\Phi)+\partial_{\mu} F_{\kappa \lambda}(\Phi)+\partial_{\lambda} F_{\mu \kappa}(\Phi)=0 \\
F_{\mu \nu}(\Phi)=\partial_{\mu} A_{\nu}(\Phi)-\partial_{\nu} A_{\mu}(\Phi) \quad, \quad A_{\mu}(\Phi)=\frac{1}{2} \omega_{a b} \Phi^{a} \partial_{\mu} \Phi^{b}
\end{array}
$$

Clearly, in eq.(12) $A_{\mu}(\Phi)$ is determined up to a $\Phi$-dependent total derivative.

From the basic definitions (22) and (31) one immediately finds that the field $F_{\mu \nu}(\Phi)$ is invariant under arbitrary field transformations (reparametrizations) $\Phi^{a}(x) \rightarrow G^{a}(\Phi(x))$ belonging to the infinite-dimensional group $\operatorname{SDiff}\left(\mathcal{T}^{2 n}\right)$, whereas its potential transforms with a $\Phi$-dependent total derivative:

$$
F_{\mu \nu}(G(\Phi))=F_{\mu \nu}(\Phi) \quad, \quad A_{\mu}(\Phi) \rightarrow A_{\mu}(\Phi)+\partial_{\mu}\left(\Gamma(\Phi)-\frac{1}{2} \Phi^{a} \frac{\partial \Gamma}{\partial \Phi^{a}}\right)
$$

Eqs.(11)-(13) naturally suggest the interpretation of $F_{\mu \nu}(\Phi)$ as an electromagnetic field strength and $A_{\mu}(\Phi)$ as the corresponding vector potential which are now composite fields 
made out of more elementary "primitive" zero-dimensional scalar fields $\Phi^{a}(x)$. Accordingly, the rôle of the ordinary local gauge invariance is now taken over by the infinite-dimensional SDiff $\left(\mathcal{T}^{2 n}\right)$ global Noether symmetry (2).

Thus, we can consider the following model of scalar "primitives" $\Phi^{a}(x)$ coupled to ordinary Dirac fermions $\psi(x)$ :

$$
\mathcal{L}=-\frac{1}{4 e^{2}} F_{\mu \nu}^{2}(\Phi)+\bar{\psi}(i \not \gamma-\not A(\Phi)-m) \psi
$$

where $F_{\mu \nu}(\Phi)$ and $A_{\mu}(\Phi)$ are given by (11) and (12), respectively, i.e., the Lagrangian (14) describes QED with special type of composite "photons". Let us stress that, although containing fourth power of derivatives on $\Phi^{a}$, the Lagrangian (14) is only second order w.r.t. time-derivatives.

The model (14), called "mini-QED", appeared previously in ref. [3 from a somewhat different motivation. Unlike ordinary QED, however, we now observe that the mini-QED model (14) does not possess local gauge invariance but rather it is invariant under infinitedimensional global volume-preserving-(symplecto-)diffeomorphic SDiff $\left(\mathcal{T}^{2 n}\right)$ Noether symmetryt :

$$
\begin{array}{r}
\Phi^{a}(x) \rightarrow G^{a}(\Phi(x)) \approx \Phi^{a}(x)+\omega^{a b} \frac{\partial \Gamma(\Phi(x))}{\partial \Phi^{b}(x)} \\
\psi(x) \rightarrow e^{\Lambda(\Phi(x))} \psi(x) \quad, \quad \Lambda(\Phi(x)) \approx \Gamma(\Phi(x))-\frac{1}{2} \Phi^{a}(x) \frac{\partial \Gamma}{\partial \Phi^{a}}
\end{array}
$$

Of course, the Lagrangian (14) is also invariant under the usual global $U(1)$ symmetry: $\psi \rightarrow e^{\alpha} \psi, \Phi^{a} \rightarrow \Phi^{a}$.

The above construction can be straightforwardly generalized for the higher " $p$-form" antisymmetric tensor gauge theories [4]. Namely, consider again a set of $s(\equiv p+1)$ zerodimensional scalar fields $\left\{\Phi^{a}(x)\right\}_{a=1}^{s}$ taking values in a smooth manifold $\mathcal{T}^{s}$. The pull-back of its canonical volume $s(\equiv p+1)$-form to Minkowski space-time gives rise to an antisymmetric $s$-tensor field strength and its associated antisymmetric $s-1$-tensor potential:

$$
\begin{array}{r}
\frac{1}{s !} \varepsilon_{a_{1} \ldots a_{s}} d \Phi^{a_{1}} \ldots d \Phi^{a_{s}}=\frac{1}{s !} F_{\mu_{1} \ldots \mu_{s}}(\Phi) d x^{\mu_{1}} \wedge \ldots \wedge d x^{\mu_{s}} \\
F_{\mu_{1} \ldots \mu_{s}}(\Phi)=\varepsilon_{a_{1} \ldots a_{s}} \partial_{\mu_{1}} \Phi^{a_{1}} \ldots \partial_{\mu_{s}} \Phi^{a_{s}} \\
F_{\mu_{1} \ldots \mu_{s}}(\Phi)=s ! \partial_{\left[\mu_{1}\right.} A_{\left.\mu_{2} \ldots \mu_{s}\right]}(\Phi) \quad, \quad A_{\mu_{1} \ldots \mu_{s-1}}(\Phi)=\frac{1}{s} \varepsilon_{a_{1} \ldots a_{s}} \Phi^{a_{1}} \partial_{\mu_{1}} \Phi^{a_{2}} \ldots \partial_{\mu_{s-1}} \Phi^{a_{s}}
\end{array}
$$

As in eq.(13), one readily verifies that the field strength (18) is invariant under arbitrary field transformations (reparametrizations) $\Phi^{a}(x) \rightarrow G^{a}(\Phi(x))$ belonging to the infinitedimensional group $\operatorname{Diff}_{0}\left(\mathcal{T}^{s}\right)(\sqrt{6})$, whereas its potential $(19)$ undergoes a $\Phi$-dependent $s-2$ rank local gauge transformation (cf. (8)-(9)) :

$$
\begin{array}{r}
A_{\mu_{1} \ldots \mu_{s-1}}(\Phi) \rightarrow A_{\mu_{1} \ldots \mu_{s-1}}(\Phi)+(s-1) ! \partial_{\left[\mu_{1}\right.} \Lambda_{\left.\mu_{2} \ldots \mu_{s-1}\right]}(\Phi) \\
\Lambda_{\mu_{1} \ldots \mu_{s-2}}(\Phi)=\left[\left(1-\frac{1}{s} \Phi^{b} \frac{\partial}{\partial \Phi^{b}}\right) \Gamma_{a_{1} \ldots a_{s-2}}+\frac{(s-3) !}{s} \Phi^{b} \frac{\partial}{\partial \Phi^{\left[a_{1}\right.}} \Gamma_{\left.|b| a_{2} \ldots a_{s-2}\right]}\right] \times \\
\times \partial_{\mu_{1}} \Phi^{a_{1}} \ldots \partial_{\mu_{s-2}} \Phi^{a_{s-2}}
\end{array}
$$

\footnotetext{
${ }^{3}$ The group parameters $\gamma_{\vec{n}}$ (cf. eq.(值)) are constant space-time independent ones.
} 
Now, similarly to mini-QED model (14) we can construct a "mini-Kalb-Ramond" model (cf. [5) using (18)-(19) for $s=3$ :

$$
\mathcal{S}=-\frac{1}{3 !} \int d^{D} x F_{\mu \nu \lambda}^{2}(\Phi(x))+\int d^{2} \sigma \frac{1}{3} \varepsilon_{a b c} \Phi^{a}(x(\sigma)) \partial_{\sigma_{1}} \Phi^{b}(x(\sigma)) \partial_{\sigma_{2}} \Phi^{c}(x(\sigma))
$$

where the second integral is over the string world-sheet given by $x^{\mu}=x^{\mu}(\sigma)$. Again as in (14), the Lagrangian (22) being of sixth order w.r.t. $\Phi^{a}$-derivatives is only quadratic w.r.t. time-derivatives.

\section{Equations of Motion and Symmetries}

First, let us consider even-dimensional space-time theories. As already pointed out in [3], in the case when the dimension of $\Phi$-target space is equal to, or greater than, the spacetime dimension, i.e., $\operatorname{dim}\left(\mathcal{T}^{2 n}\right) \equiv 2 n \geq D$, any vector potential can be represented (in a non-unique way) in the form (12). This is not any more true when $\operatorname{dim}\left(\mathcal{T}^{2 n}\right) \leq D-2$, since then the topological density $\varepsilon^{\mu_{1} \mu_{2} \ldots \mu_{D}} F_{\mu_{1} \mu_{2}}(\Phi) \cdots F_{\mu_{D-1} \mu_{D}}(\Phi)$, and even the associated topological Chern-Simons current, are identically zero :

$$
\begin{array}{r}
\varepsilon^{\mu_{1} \mu_{2} \ldots \mu_{D}} A_{\mu_{2}}(\Phi) F_{\mu_{3} \mu_{4}}(\Phi) \cdots F_{\mu_{D-1} \mu_{D}}(\Phi)= \\
\frac{1}{2} \omega_{a_{1}\left[a_{2}\right.} \ldots \omega_{\left.a_{D-1} a_{D}\right]} \varepsilon^{\mu_{1} \mu_{2} \ldots \mu_{D}} \Phi^{a_{1}} \partial_{\mu_{2}} \Phi^{a_{2}} \partial_{\mu_{3}} \Phi^{a_{3}} \cdots \partial_{\mu_{D}} \Phi^{a_{D}}=0 \quad(\text { for } 2 n \leq D-2)
\end{array}
$$

Here again the square brackets indicate total antisymmetrization of indices. Thus, as emphasized in [3], mini-QED with a target space of the scalar "primitives" satisfying the condition $\operatorname{dim}\left(\mathcal{T}^{2 n}\right) \leq D-2$ does not exhibit the usual axial anomalies.

Similar phenomena occur in odd space-time dimensions $D$. Namely, when $\operatorname{dim}\left(\mathcal{T}^{2 n}\right) \geq$ $D+1$ any vector potential can be represented (in a non-unique way) in the form (12), whereas when $\operatorname{dim}\left(\mathcal{T}^{2 n}\right) \leq D-1$ the topological Chern-Simons term is identically zero.

The equations of motion for (14) are:

$$
\begin{aligned}
\partial_{\mu} \Phi^{a}\left[\frac{1}{e^{2}} \partial_{\nu} F^{\mu \nu}(\Phi)+\bar{\psi} \gamma^{\mu} \psi\right] & =0 \\
(i \partial-\not \mathcal{A}(\Phi)-m) \psi & =0
\end{aligned}
$$

The infinite set of Noether currents corresponding to the SDiff $\left(\mathcal{T}^{2 n}\right)$ symmetry of (14) reads:

$$
\begin{array}{r}
J^{\mu}[f]=f(\Phi)\left[\frac{1}{e^{2}} \partial_{\nu} F^{\mu \nu}(\Phi)+\bar{\psi} \gamma^{\mu} \psi\right] \\
\partial_{\mu} J^{\mu}[f]=0 \quad, \quad \delta_{\Gamma} J^{\mu}[f]=J^{\mu}[\{f, \Gamma\}]
\end{array}
$$

where $f(\Phi)$ is an arbitrary (smooth) function on the $\Phi$-target space $\mathcal{T}^{2 n}$ and $\delta_{\Gamma}$ indicates an infinitesimal symplecto-diffeomorphic transformation (15)-(16). In the particular case of torus target space (4) we have:

$$
J^{\mu}[f]=\sum_{\vec{n}} f_{\vec{n}} J_{\vec{n}}^{\mu} \quad, \quad J_{\vec{n}}^{\mu}=e^{i \vec{n} . \vec{\Phi}}\left[\frac{1}{e^{2}} \partial_{\nu} F^{\mu \nu}(\Phi)+\bar{\psi} \gamma^{\mu} \psi\right]
$$


The first thing one notices in eqs.(24)-(25) is that the $\Phi$-equations of motion differ from the usual Maxwell equations (with a "composite" vector potential) just by the contraction of the latter with the $2 n \times D$ matrix $\left\|\partial_{\mu} \Phi^{a}\right\|$. This observation together with eq.(23) lead us to the following:

Main statement (even D). The $\Phi$-equations of motion (24) coincide with the Maxwell ones if and only if $\operatorname{rank}\left\|\partial_{\mu} \Phi^{a}\right\| \geq D$ (cf. eq.(23)). This implies that both the topological Chern-Simons current as well as its divergence (the topological density) are non-zero. Alternatively, the $\Phi$-equations of motion (24) possess non-Maxwell solutions if either the topological Chern-Simons current or the topological density are zero, i.e., rank $\left\|\partial_{\mu} \Phi^{a}\right\| \leq D-2$ (cf. eq.(23)).

Completely analogous statement holds for odd space-time dimensions $D$ upon substituting $D \rightarrow D+1$ above.

Therefore, in the sequel we shall be interested in the second case, i.e., the case with zero topological Chern-Simons current, which implies the existence of a non-Maxwell sector of mini-QED (14). In particular, when $\operatorname{dim}\left(\mathcal{T}^{2 n}\right) \equiv 2 n \leq D-2$ mini-QED is generically nonMaxwell. It can be viewed as a truncation of a model proposed in [8] describing ordinary Maxwell $D=4$ QED coupled to a pseudo-scalar "pion" field $\phi$ :

$$
\mathcal{L}(\psi, A ; \phi)=-\frac{1}{4 e^{2}} F_{\mu \nu}^{2}+\bar{\psi}(i \not \partial-\not A-m) \psi+\frac{1}{2} \phi \varepsilon^{\mu \nu \kappa \lambda} F_{\mu \nu} F_{\kappa \lambda}+\mathcal{L}(\phi)
$$

where $\mathcal{L}(\phi)$ contains the kinetic and self-interacting terms for the "pion". Ignoring in (29) the purely "pionic" Lagrangian, e.g., considering the heavy mass limit for $\phi$, we see that the field $\phi$ becomes a non-dynamical Lagrange multiplier enforcing the constraint for zero topological density. Thus, mini-QED is a particular realization of the heavy mass limit of the model (29) with the additional condition for a vanishing topological Chern-Simons current.

\section{Physical Consequences: New Effects}

For definiteness, from now on we shall consider mini-QED (14) in ordinary $D=4$ space-time and with a two-dimensional $\Phi$-target space.

\subsection{Magneto-Hydrodynamical Analogy}

The existence of non-Maxwell solutions to (24) means that there exists a non-zero additional current $\mathcal{J}^{\mu}(\Phi)$ - suitable functional of $\Phi^{a}$, such that:

$$
\frac{1}{e^{2}} \partial_{\nu} F^{\mu \nu}(\Phi)+\bar{\psi} \gamma^{\mu} \psi+\mathcal{J}^{\mu}(\Phi)=0 \quad, \quad \partial_{\mu} \Phi^{a} \mathcal{J}^{\mu}(\Phi)=0
$$

The second eq.(30) implies also:

$$
A_{\mu}(\Phi) \mathcal{J}^{\mu}(\Phi)=0 \quad, \quad F_{\lambda \mu}(\Phi) \mathcal{J}^{\mu}(\Phi)=0
$$

Using the standard three-dimensional Maxwell notations, one can rewrite the second eq.(31) as:

$$
\begin{array}{r}
\vec{E}(\Phi) \cdot \overrightarrow{\mathcal{J}}(\Phi)=0 \\
-\mathcal{J}^{0}(\Phi) \vec{E}(\Phi)+\vec{B}(\Phi) \times \overrightarrow{\mathcal{J}}(\Phi)=0
\end{array}
$$


From (33) one easily verifies that non-zero solutions for $\mathcal{J}^{\mu}(\Phi)$ exist if $\vec{E}(\Phi) . \vec{B}(\Phi)=0$ which is precisely the expression for zero topological density $\varepsilon^{\mu \nu \kappa \lambda} F_{\mu \nu}(\Phi) F_{\kappa \lambda}(\Phi)$. Then, eq.(33) can be represented in the following "magneto-hydrodynamical" form (cf. [9]) :

$$
\vec{E}(\Phi)+\frac{\overrightarrow{\mathcal{J}}(\Phi)}{\mathcal{J}^{0}(\Phi)} \times \vec{B}(\Phi)=0
$$

where $\vec{v}(\Phi) \equiv \overrightarrow{\mathcal{J}}(\Phi) / \mathcal{J}^{0}(\Phi)$ can be viewed as a $\Phi$-dependent velocity field. For such velocity, however, $|\vec{v}|$ may exceed 1 . In any case, the condition $\vec{E}(\Phi) \cdot \vec{B}(\Phi)=0$ guarantees that there is always a frame where either the electric field is zero (as in usual magnetohydrodynamics) or where the magnetic field is zero ("dual magnetohydrodynamics").

\subsection{Electromagnetic Vacuum in Mini-QED}

Now, let us consider the mini-QED electron dynamics in the presence of electromagnetic vacuum:

$$
F_{\mu \nu}\left(\Phi_{v a c}\right)=0
$$

The general solution to (35) can be taken in the form: $\Phi_{v a c}^{1}(x) \equiv u(x), \Phi_{v a c}^{2}(x)=B(u(x))$ - arbitrary local function of $u$. Then eqs.(25)-(24) become (after an appropriate phase transformation of the Dirac field) :

$$
\begin{aligned}
(i \not \partial-m) \psi_{v a c} & =0 \\
\left(\bar{\psi}_{v a c} \gamma^{\mu} \psi_{v a c}\right) \partial_{\mu} u & =0
\end{aligned}
$$

The last equation means that $\Phi_{v a c}^{a}(x)$ are constant along the straight free-electron world-lines, i.e., $\frac{\partial}{\partial \tau} \Phi_{v a c}^{a}(x(\tau))=0$. Thus, one gets usual plain-wave solutions without the self-energy problem of ordinary QED (where one would get that the current must vanish simultaneously).

\subsection{Gauge-Invariant Photon Mass Generation}

Let us now show that mini-QED built out of two primitive scalars allows propagation of massive gauge-invariant modes in $2+1$ and in $3+1$ dimensions.

First, let us consider the $2+1$-dimensional case. The basic observation is that the equations of motion for mini-QED in the absence of sources:

$$
\partial_{\mu} \Phi^{a} \partial_{\nu} F^{\mu \nu}(\Phi)=0
$$

allow, apart from the pure Maxwell solutions where $\partial_{\nu} F^{\mu \nu}(\Phi)=0$, also non-Maxwell solutions of the following form:

$$
\partial_{\nu} F^{\mu \nu}(\Phi)=\mu(\Phi) F^{* \mu}(\Phi) \quad, \quad F^{* \mu}(\Phi)=\frac{1}{2} \varepsilon^{\mu \nu \alpha} F_{\nu \alpha}(\Phi)
$$

Consistency of (39) with the "free" mini-QED eqs.(38) is easily verified remembering the form of $F^{\mu \nu}(\Phi)=\varepsilon_{a b} \partial_{\mu} \Phi^{a} \partial_{\nu} \Phi^{b}$. Indeed, substituting (39) in the l.h.s. of (38) one gets:

$$
\partial_{\mu} \Phi^{a} \partial_{\nu} F^{\mu \nu}(\Phi)=\frac{1}{2} \mu(\Phi) \varepsilon^{\mu \nu \alpha} F_{\nu \alpha}(\Phi) \partial_{\mu} \Phi^{a}=\frac{1}{2} \mu(\Phi) \varepsilon^{\mu \nu \alpha} \varepsilon_{b c} \partial_{\mu} \Phi^{[a} \partial_{\nu} \Phi^{b} \partial_{\alpha} \Phi^{c]}=0
$$


where the last equality follows from antisymmetrization of three indices taking only two values.

Furthermore, taking the divergence of eq.(39) on both sides we obtain:

$$
\partial_{\mu} \partial_{\nu} F^{\mu \nu}(\Phi)=0=\frac{\partial \mu(\Phi)}{\partial \Phi^{a}} \partial_{\mu} \Phi^{a} F^{* \mu}(\Phi)
$$

This is consistent since, as we showed in (40), $\partial_{\mu} \Phi^{a} F^{* \mu}(\Phi)=0$. In the case where $\mu(\Phi)=$ $\mu=$ const , eq.(39) coincides with the equation of the topologically massive Chern-Simons theory (see [10, 11] and references therein). However, the solution (39) to mini-QED eqs.(38) exhibits an interesting new feature, namely that here the mass is not determined, i.e., it could have any value.

The solutions of eq.(39) for the case $\mu(\Phi)=\mu=$ const are known [11]. As it has been shown in ref. [11], the field strengths $F_{0 i}=E_{i}$ and $B=\varepsilon_{i j} \partial_{i} A_{j}$ can be expressed in terms of a massive free field $\varphi$ satisfying $\left(\square+\mu^{2}\right) \varphi=0$ according to:

$$
E_{i}=\varepsilon_{i j} \hat{\partial}_{j} \dot{\varphi}+\mu \hat{\partial}_{j} \varphi \quad, \quad B=-\sqrt{-\nabla^{2}} \varphi \quad, \quad \hat{\partial}_{j}=\frac{\partial_{j}}{\sqrt{-\nabla^{2}}}
$$

where the dot indicates $\partial_{0}$-derivative and $i, j=1,2$.

It is of interest to see how the solutions (42) can be expressed in terms of the primitive scalar fields $\Phi^{a}$, i.e., $E_{i}(\Phi)=\varepsilon_{a b} \dot{\Phi}^{a} \partial_{i} \Phi^{b}$ and $B(\Phi)=\frac{1}{2} \varepsilon^{i j} \varepsilon_{a b} \partial_{i} \Phi^{a} \partial_{j} \Phi^{b}$. For this purpose, let us consider $E_{i}$ and $B$ (42) given in terms of $\varphi$ which is a simple plane wave of the form $\varphi=A \cos \left(k^{0} x^{0}-k^{i} x^{i}\right)$, namely:

$$
\begin{aligned}
& \hat{\partial}_{i} \varphi=-A \hat{k^{i}} \sin \left(k^{0} x^{0}-k^{i} x^{i}\right) \quad, \quad \hat{\partial}_{i} \dot{\varphi}=-A \hat{k^{i}} k^{0} \cos \left(k^{0} x^{0}-k^{i} x^{i}\right) \\
& B=-A \sqrt{\vec{k}^{2}} \cos \left(k^{0} x^{0}-k^{i} x^{i}\right)
\end{aligned}
$$

Let us consider the rest frame, that is the limit $k^{i} \longrightarrow 0$. In such a case we obtain (since $\left.k^{0} \longrightarrow \mu\right):$

$$
E_{i}=-A \mu\left[\varepsilon_{i j} \hat{k}_{j} \sin (\mu t)+\hat{k}_{i} \cos (\mu t)\right] \quad, \quad B=0
$$

We see that when $k^{i} \longrightarrow 0$ the unit vector $\hat{k}_{i}$ does not disappear from the expression for the electric field in the rest frame (44). Such a unit vector $\hat{k}_{i}$ can be arbitrarily chosen in any space direction. For example, choosing $\hat{k}_{i}$ in the $x$-direction implies that $\epsilon_{i j} \hat{k}_{j}$ is in the $y$-direction. Therefore, the electric field described by eq.(44) is a rotating one in the $x-y$ plane. The effect of choosing $\hat{k}_{i}$ along a different space direction is equivalent to a time shift in eq.(44).

A choice for $\Phi^{1}$ and $\Phi^{2}$ that reproduces the configuration (44) is:

$$
\Phi^{1}=-t \mu \quad, \quad \Phi^{2}=A x^{i}\left[\varepsilon_{i j} \hat{k}_{j} \sin (\mu t)+\hat{k}_{i} \cos (\mu t)\right]
$$

The generalization of (45) to an arbitrary coordinate system where the $\varphi$ - "particle" has an arbitrary momentum reads:

$$
\Phi^{1}=-k_{\nu} x^{\nu} \quad, \quad \Phi^{2}=x_{\nu}\left[\varepsilon^{\nu \alpha \beta} s_{\alpha} k_{\beta} \sin \left(k_{\sigma} x^{\sigma}\right)+\mu s^{\nu} \cos \left(k_{\sigma} x^{\sigma}\right)\right]
$$


where $s^{\nu}$ is any vector satisfying $s^{\nu} k_{\nu}=0$, i.e., it is such that in the rest frame $s^{\nu}=$ $(0, \vec{s})$. Thus, eqs.(46) provide a solution to the sourceless mini-QED equations of motion (38) describing gauge invariant massive photons with an arbitrary mass.

The $2+1$-dimensional non-Maxwell mini-QED solution given above can be easily embedded in the $3+1$-dimensional mini-QED given by (38). In this case we have to select arbitrarily one space dimension which we do not want to appear in the solution, let us say $z$. Then, instead of (39) we should use:

$$
\partial_{\nu} F^{\mu \nu}(\Phi)=\frac{1}{2} \mu(\Phi) \varepsilon^{3 \mu \nu \alpha} F_{\nu \alpha}(\Phi)
$$

where there is no dependence of any field on $z$.

The physical consequences of a continuous mass spectrum, similar to the one found here, have been discussed in ref. [12].

\section{Open Problems and Outlook; Mini-QED as an Induced Massive Gravity}

The next most important question is quantization of the mini-QED model (14). Applying formally the general arguments of renormalization theory [13, namely that the possible counterterms canceling the ultra-violet divergences must be local, covariant functionals of the constituent fields of dimension $D=4$ and preserving the global Noether SDiff $\left(\mathcal{T}^{2 n}\right)$ symmetry, one easily deduces that these counterterms will be exactly the same as in ordinary QED, i.e., the renormalized mini-QED Lagrangian will be of the same form as (14) with the usual charge-, mass- and multiplicative field renormalizations.

The problem with (14) is, however, that the kinetic term for the scalar "primitives" $\Phi^{a}$ is of a non-standard quartic form which does not allow to develop the usual Feynman diagram expansion. Moreover, if we would add to (14) an ordinary kinetic term for $\Phi^{a}$, the latter would break the SDiff $\left(\mathcal{T}^{2 n}\right)$ symmetry. Obviously, we have to rewrite (14) in an equivalent form with the help of auxiliary fields which would bilinearize the $\Phi^{a}$-part of the Lagrangian - much in the same way one deals with models having four-fermion interaction terms.

Considering again for simplicity two-dimensional $\Phi$-target space and using the simple identity:

$$
\varepsilon_{a b} \varepsilon_{c d}=\delta_{a c} \delta_{b d}-\delta_{a d} \delta_{b c}
$$

one can represent the first "Maxwell" term in (14) as follows:

$$
F_{\mu \nu}^{2}(\Phi)=-\left(\partial_{\mu} \Phi^{a} \partial_{\nu} \Phi_{a}\right)\left[\eta^{\mu \kappa} \eta^{\nu \lambda}-\eta^{\mu \nu} \eta^{\kappa \lambda}\right]\left(\partial_{\kappa} \Phi^{b} \partial_{\lambda} \Phi_{b}\right)
$$

where $\eta^{\mu \nu}$ is the flat Minkowski space-time metric tensor. Taking into account (49), the mini-QED Lagrangian (14) acquires the following equivalent form:

$$
\mathcal{L}=\frac{1}{2} h^{\mu \nu} \partial_{\mu} \Phi^{a} \partial_{\nu} \Phi_{a}-\frac{e^{2}}{4} h^{\mu \nu}\left(\eta^{\mu \kappa} \eta^{\nu \lambda}-\frac{1}{3} \eta^{\mu \nu} \eta^{\kappa \lambda}\right) h^{\kappa \lambda}-\left(\bar{\psi} \gamma^{\mu} \psi\right) \frac{1}{2} \varepsilon_{a b} \Phi^{a} \partial_{\mu} \Phi^{b}+\bar{\psi} i \not \partial \psi
$$

Here $h^{\mu \nu}$ is an auxiliary symmetric tensor field classically given by:

$$
h^{\mu \nu}=\frac{1}{e^{2}}\left[\partial_{\mu} \Phi^{a} \partial_{\nu} \Phi_{a}-\eta^{\mu \nu}\left(\partial_{\lambda} \Phi^{b} \partial^{\lambda} \Phi_{b}\right)\right]
$$


The first term in eq.(50) indicates that $h^{\mu \nu} \equiv \sqrt{-g} g^{\mu \nu}$ can be viewed as composite gravitational field (cf. (51)) coupled to the scalar primitives $\Phi^{a}$ and being massive (cf. the second term in (50)) with a mass proportional to the square of the electric charge. Integrating out the $\Phi^{a}$-fields will produce (upon neglecting the fermions) the standard quantum effective action of scalar fields in a curved background |14 with the usual divergent cosmological, Einstein- and $R^{2}$-terms for $h^{\mu \nu} \equiv \sqrt{-g} g^{\mu \nu}$. Thus, we find an intriguing equivalence of the mini-QED model (14) with a theory of purely induced and massive gravity (50) which, however, is formally non-renormalizable. On the other hand, unlike ordinary gravity (with dimensionless $h^{\mu \nu} \equiv \sqrt{-g} g^{\mu \nu}$ ) the field $h^{\mu \nu}$ in (50) has dimension 2 (cf. (51)) and, therefore, the usual divergent non-renormalizable semi-classical expansion of ordinary gravity interacting with matter fields around flat background metric is not appropriate for mini-QED in the form (50). Obviously, a bilinearization of mini-QED (14) different from (50) is needed for its proper quantization - a question, which is currently under investigation.

Another possible venue is to exploit the Ward identities for the infinite-dimensional global Noether SDiff $\left(\mathcal{T}^{2 n}\right)$ symmetry [15] to obtain non-perturbative information for the quantum correlation functions of mini-QED. In particular, an interesting important question arises about possible quantum deformation of the classical Noether SDiff $\left(\mathcal{T}^{2 n}\right)$ symmetry, e.g., the area-preserving-diffeomorphisms' $\operatorname{SDiff}\left(\mathcal{T}^{2}\right)$ symmetry might acquire central extension (5) and/or be deformed into (centrally extended) $\mathbf{W}_{\mathbf{1}+\infty}$-symmetry, as it happens in $D=2$ conformal field theory (cf., e.g. [6]).

Acknowledgements. E.N. and S.P. gratefully acknowledge support from the Ben-Gurion University, Beer-Sheva. E.N. is also thankful to H. Aratyn for cordial hospitality at the University of Illinois at Chicago where this work was completed.

\section{References}

[1] L. Faddeev and L. Takhtajan, "Hamiltonian Methods in the Theory of Solitons", Springer (1987); L. Dickey, "Soliton Equations and Hamilton Systems", World Sci. (1991); N. Bogoliubov, A. Izergin and V. Korepin, "Quantum Inverse Scattering Method and Correlation Functions", Cambridge Univ. Press (1993)

[2] V. Arnold, "Mathematical Methods of Classical Mechanics", Springer (1989)

[3] E.I. Guendelman, preprint $B G U-95 / 9 / A p r-P H$

[4] M. Henneaux and C. Teitelboim, in "Between Quantum and Cosmos", Studies and Essays in Honor of J.A. Wheeler, Princeton Univ. Press (1988)

[5] M. Kalb and P. Ramond, Phys. Rev. D9 (1974) 2273

[6] I. Bakas and E. Kiritsis, Int. Journ. Mod. Phys, A7 [Suppl. 1A] (1992) 55; C. Pope, Lectures on $W$-Algebras and $W$-Gravity, in High Energy Physics and Cosmology, Proc. of Trieste Summer School, 1991 (also in hep-th/9112078)

[7] E. Floratos and J. Iliopolus, Phys. Lett. 201B (1988) 237; D. Fairlie, P. Fletcher and C. Zachos, Phys. Lett. 218B (1989) 203 
[8] E.I. Guendelman and D. Owen, Phys. Lett. 251B (1990) 439; Phys. Lett. 252B (1990) 113

[9] L. Landau and E. Lifshitz, "Electrodynamics of Continuous Media", (Course on Theor. Phys., vol.8), Pergamon (1984); J. Jackson, "Classical Electrodynamics", Second Edition, Wiley $(1975)$

[10] S. Deser, R. Jackiw and S. Templeton, Ann. of Phys. 140 (1982) 372; R. Jackiw, in "Relativity and Topology" (Les Houches, 1983), North Holland (1984)

[11] R. Jackiw, in "Asymptotic Realms of Physics", A. Guth, K. Huang and R. Jaffe, eds., MIT Press (1983)

[12] B. McCoy and T.T. Wu, Phys. Lett. 72B (1977) 219

[13] N. Bogoliubov and D. Shirkov, "Introduction to the Theory of Quantized Fields", Wiley (1980); O. Zavyalov, "Renormalized Quantum Field Theory", Kluwer (1990)

[14] N. Birrel and P. Davies, "Quantum Fields in Curved Space-Time" Cambridge Univ. Press (1982)

[15] H. Aratyn, E. Nissimov and S. Pacheva, Phys. Lett. 255B (1991) 359; E. Nissimov, S. Pacheva and I. Vaysburd, Phys. Lett. 284B (1992) 254 